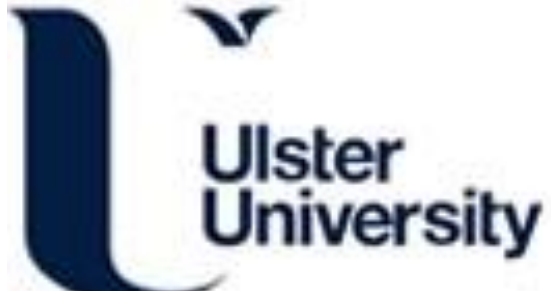

\section{Camera Phone-Based Quantitative Analysis of C-Reactive Protein ELISA}

McGeough, C., \& O'Driscoll, S. (2013). Camera Phone-Based Quantitative Analysis of C-Reactive Protein ELISA. IEEE Transactions on Biomedical Circuits and Systems, pp, 1.

https://doi.org/10.1109/TBCAS.2012.2234122

Link to publication record in Ulster University Research Portal

Published in:

IEEE Transactions on Biomedical Circuits and Systems

Publication Status:

Published (in print/issue): 01/01/2013

DOI:

10.1109/TBCAS.2012.2234122

\section{Document Version}

Author Accepted version

\section{General rights}

Copyright for the publications made accessible via Ulster University's Research Portal is retained by the author(s) and / or other copyright owners and it is a condition of accessing these publications that users recognise and abide by the legal requirements associated with these rights.

\section{Take down policy}

The Research Portal is Ulster University's institutional repository that provides access to Ulster's research outputs. Every effort has been made to ensure that content in the Research Portal does not infringe any person's rights, or applicable UK laws. If you discover content in the Research Portal that you believe breaches copyright or violates any law, please contact pure-support@ulster.ac.uk. 


\title{
Camera Phone-Based Quantitative Analysis of C-Reactive Protein ELISA
}

\author{
Cathy M. McGeough and Stephen O'Driscoll, Member, IEEE
}

\begin{abstract}
We demonstrate the use of a camera phone as a lowcost optical detector for quantitative analysis of a high-sensitivity C-reactive protein (hs-CRP) enzyme-linked immunosorbent assay (ELISA). The camera phone was used to acquire images of the ELISA carried out in a conventional 96 well plate. Colorimetric analysis of the images was used to determine a standard curve that exhibited excellent agreement with a fitted 4-parameter logistic model $\left(R^{2}=0.998\right)$. The limit of detection (LOD) for this approach was determined to be $0.026 \pm 0.002 \mu \mathrm{g} / \mathrm{ml}(1.035 \pm$ $0.079 \mu \mathrm{M}$ ) CRP. Furthermore, these results were found to be in very close agreement with measurements obtained for the same assay using a laboratory-based instrument. These findings indicate the basic technology to enable low-cost quantitative home-based monitoring of an important clinical biomarker of inflammatory disease may already be present in the patient's home.
\end{abstract}

Index Terms-Camera phone, C-reactive protein, enzyme-linked immunosorbent assay (ELISA), inflammatory disease, quantitative biomarker detection.

\section{INTRODUCTION}

$\mathbf{T}$ HERE is growing recognition among healthcare stakeholders that patient-centric models of healthcare delivery are both highly cost-effective and beneficial to the patient. In particular, home-based self-monitoring is now a tangible reality for individuals with adequately controlled or stable chronic disease. In such applications, clinical management requires quantitative biomarker information in order to accurately monitor and assess treatment progress. Currently, most testing for biomarkers is carried out in centralised laboratories or at the point-of-care (e.g., doctor's clinic) using specialised techniques and measurement equipment. One such technique is the enzyme-linked immunosorbent assay (ELISA). Typically, ELISAs are carried out in well plates that are analyzed optically using expensive plate reader instruments that employ spectrophotometric techniques to determine biomarker

Manuscript received May 29, 2012; revised October 26, 2012; accepted November 29, 2012. This material is based in part upon works supported by Enterprise Ireland under Grant PC/2008/0133 and the European Regional Development Fund (ERDF) under the National Strategic Reference Framework (NSRF) 2007-2013. This paper was recommended by Associate Editor R. Etienne-Cummings.

C. M. McGeough was with the Biomedical Diagnostics Institute, Dublin City University, Glasnevin, Dublin 9, Ireland. She is now with Stratified Medicine, Biomedical Sciences Research Institute, University of Ulster, Coleraine BT52 1SA, Northern Ireland (e-mail: c.mcgeough@ulster.ac.uk).

S. O'Driscoll is with the Biomedical Diagnostics Institute, Dublin City University, Glasnevin, Dublin 9, Ireland (e-mail: stephen.odriscoll@dcu.ie).

Color versions of one or more of the figures in this paper are available online at http://ieeexplore.ieee.org.

Digital Object Identifier 10.1109/TBCAS.2012.2234122 concentration. However, to fully realize the potential benefits of home-based self-monitoring of disease it will be necessary to develop alternative low-cost technologies that enable the detection and quantification of biomarkers in non-traditional settings such as the home. In this context, the ability to develop low-cost ELISA-based diagnostic tests has significant potential and a number of parallel approaches have been reported that address the development of alternative low-cost optical detection strategies [1]-[3] and assay platforms [4]-[8] for this purpose. However, the ability to leverage the ubiquitous nature of camera phone technology as an optical detection platform for analytical applications in healthcare has transformative potential.

Camera phones have been used in a number of analytical applications to acquire images that can be analyzed, either in-situ (i.e., by the phone) or remotely, in order to determine quantitative information. For example, camera phones have been demonstrated to enable: in-situ quantitative assessment of UV exposure in solar disinfection of water [9], real-time detection of trace gases and contaminants [10], [11], detection of biomarkers using lateral flow strips [12] and other novel diagnostic tests [13].

With $50-80 \%$ of global health spending related to chronic disease [14], there is clear impetus for the development of more efficient and cost-effective methods of disease management. The use of camera phones as a low-cost health-enabling technology to support self-monitoring of chronic disease may be one such approach. However, adaptation will depend on their ability to detect biomarkers relevant to the management of chronic disease.

Inflammation is the underlying basis of a number of important chronic diseases including cardiovascular disease (CVD), rheumatoid arthritis (RA) and inflammatory bowel disease (IBD). C-reactive protein (CRP) is one of the most widely used biomarkers of inflammation. CRP is an acute phase reactant that is released from the liver during an inflammatory response to tissue injury, infection, malignancy and autoimmunity. The blood concentration of CRP is routinely used to indicate the degree of inflammation in chronic inflammatory diseases such as CVD [15], RA [16], Crohn's disease [17] and psoriasis [18]. More recently, the utility of high-sensitivity CRP (hs-CRP) assays, which are capable of detecting very low concentrations, has been highlighted both in rheumatology as a means of assessing clinical remission $(<10 \mu \mathrm{g} / \mathrm{ml})$ [16] and in the assessment of CVD risk $(<1 \mu \mathrm{g} / \mathrm{ml}$ - low, $1-3 \mu \mathrm{g} / \mathrm{ml}$ —average, $>3 \mu \mathrm{g} / \mathrm{ml}$ - high) [15]. In this work, we demonstrate the use of camera phone imaging in the quantitative analysis of a hs-CRP ELISA. This work may have significant implications for the development of low-cost measurement technologies that 
enable home-based self-monitoring of a number of significant chronic inflammatory diseases.

\section{EXPERIMENTAL}

\section{A. High-Sensitivity C-Reactive Protein ELISA}

The hs-CRP ELISA was carried out in a standard 96 well plate using three assay replicates of 7 known concentrations (including blank) and one unknown sample for testing. These known concentrations are used to determine a standard (i.e., calibration) curve. The assay was performed by first adding $100 \mu \mathrm{l}$ of $5 \mu \mathrm{g} / \mathrm{ml}$ rabbit anti-human CRP capture antibody to each well and incubating for $1 \mathrm{hr}$ at $37^{\circ} \mathrm{C}$. The plate was blotted to remove unbound antibody and blocked with $200 \mu \mathrm{l}$ of 5\% BSA in PBS ( $\mathrm{pH} \mathrm{7.4)} \mathrm{for} 1 \mathrm{hr}$ at $37^{\circ} \mathrm{C}$ (Sigma, Ireland). The plate was then washed 3 times with PBS. CRP antigen (Hytest, Finland) concentrations were prepared in PBS $(0-5 \mu \mathrm{g} / \mathrm{ml})$ and incubated on the plate for $1 \mathrm{hr}$ at $37^{\circ} \mathrm{C}$. The plate was washed 5 times with PBST $(0.05 \%$ Tween) and PBS before the addition of $100 \mu \mathrm{l}$ per well of $10 \mu \mathrm{g} / \mathrm{ml}$ detection antibody C5 (Hytest, Finland) for one hour at $37^{\circ} \mathrm{C}$. The plate was washed a further five times with PBST and PBS. $100 \mu \mathrm{l}$ of 1:1000 conjugate anti-mIgG-HRP (Sigma, Ireland) was added to each well and incubated for 1 hour at $37^{\circ} \mathrm{C}$. The wells were washed 5 more times with PBST and PBS before the addition of $50 \mu \mathrm{l} \mathrm{TMB} \mathrm{en-}$ zyme substrate (Sigma, Ireland). The plate was left to develop for 10 minutes and images of the plate were obtained with the camera phone.

\section{B. Camera Phone Image Acquisition and Analysis}

The camera phone used in this work was a Nokia N96 smartphone which employs an 8-bit $2592 \times 1944$ pixel RGB image sensor. To provide a uniform background for the images, a sheet of plain white paper was placed beneath the well plate. The phone was positioned directly above the well plate at a distance of approx. $12 \mathrm{~cm}$. The large number of pixels available in this image sensor provides high spatial sampling of the image which is advantageous for this type of application where the smartphone is placed at a distance from the object of interest (i.e., well plate). To reduce shadowing caused by the phone and overhead lighting, a standard desk lamp (40 W bulb) was located above the well plate and inclined at an angle of 50 degrees to the surface of the bench top. A total of five images were acquired using the phone's camera and stored in memory. The camera's flash was disabled during image acquisition. To assess the camera phone-based method, we compared its performance to that of a standard analysis of the well plate carried out using a laboratory plate reader. For this standard analysis, the reaction was stopped immediately after acquisition of the camera phone images by adding $50 \mu \mathrm{l}$ of $0.1 \mathrm{M}$ hydrochloric acid $(\mathrm{HCl})$ to each well. The plate was then placed in the plate reader (TECAN Infinite M200, TECAN, Switzerland) and the sample absorbance at $450 \mathrm{~nm}$ measured. The absorbance values obtained for a given concentration were then averaged and the standard deviation determined. The addition of $\mathrm{HCl}$ results in each of the samples turning yellow. However, the camera phone

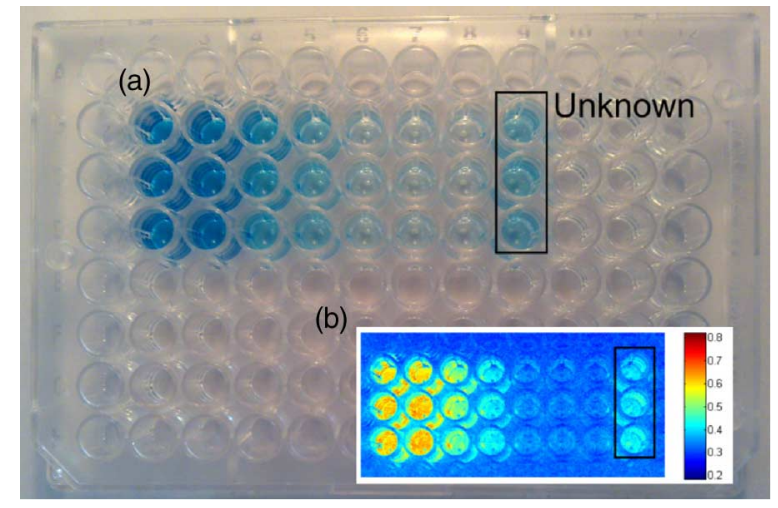

Fig. 1. Representative image of standard 96 well plate acquired by mobile phone (cropped for display purposes). (a) Assay region of well plate. (b) $b$-channel image of assay region.

images were acquired prior to the addition of $\mathrm{HCl}$ hence the solutions remains blue. Fig. 1(a) shows a representative image of the well plate obtained using the camera. Each assay replicate occupies a separate row of the plate with sample concentration decreasing from left-to-right. The second last well in each row contains the blank while the last contains the unknown sample. The images were then transferred from the camera phone to a personal computer and analysed using custom-software developed in the MATLAB (MathWorks Inc.) environment. The images were then transformed from the native camera RGB color space to the normalised RGB $(r g b)$ color space to reduce the effect of variations in illumination intensity [19]. The normalised blue channel ( $b$-channel) was selected for all subsequent image analysis. The decision was based on the analysis detailed in Section II-C. Fig. 1(b) shows the assay region of the corresponding $b$-channel image shown in Fig. 1(a). Following transformation, the average of a sample of $\mathrm{N}=121$ pixels $(11 \times 11$ pixel region) from each well was calculated. Since the sample volume in each well was constant, this average response was used as a measure of analyte concentration. This procedure was repeated for each well in all images. The responses for corresponding concentrations within each image for each replicate were then averaged to produce a $3 \times 8$ (replicate $\times$ sample) matrix. The calibration data were determined by averaging the columns of this matrix. The standard deviation of these values was used as a measure of inter-replicate variability.

\section{Color Channel Selection}

Our motivation for acquiring images prior to the addition of $\mathrm{HCl}$ was based on an analysis of the camera's color detection performance. A challenge associated with the use of imaging devices that implement the RGB color model is that this color model is device dependent [20]. Consequently, it is necessary to evaluate which of the three color channels provides the best detection performance for a particular measurement application since the manner in which color is detected can change from one device to another.

To do this, we performed a number of experiments in which images of the assay were acquired before and after the addition of $\mathrm{HCl}$. Each of the normalised color channels (i.e., $r, g$ 
TABLE I

COLOR CHANNEl ANALYSIS

\begin{tabular}{lcccc}
\hline \hline Assay & Channel & Range & $\sigma_{\mathrm{Max}}$ & Range/ $\sigma_{\text {Max }}$ \\
\hline Stopped & red & 0.133 & 0.017 & 7.896 \\
& green & 0.120 & 0.020 & 6.071 \\
& blue & 0.253 & 0.035 & 7.217 \\
\hline Unstopped & red & 0.223 & 0.025 & 8.765 \\
& green & 0.065 & 0.006 & 10.464 \\
& blue & 0.273 & 0.015 & 18.691 \\
\hline \hline
\end{tabular}

and $b$ ) were analysed in the manner described previously. Based on these response data, the range (i.e., difference between maximum and minimum response) and maximum uncertainty (estimated as the standard deviation) for each channel was determined. The ratio of the range and the uncertainty was calculated and used as an estimate of the each channel's signal-to-noise ratio (SNR). Table I shows representative results of this analysis which indicates that the $b$-channel, when used in conjunction with an assay that is not stopped, is optimal in terms of SNR (18.691).

In addition to this measure, it is also important to consider the nature of the channel response to changes in analyte concentration. As stated previously, the image sensor used in the camera phone is an RGB sensor. The RGB color model is an additive color model that represents/detects color as a summation of the three primary color components. This model is device dependent which means that different devices will represent the same color differently. For this application, the manner in which color is detected can have significant implications for an assessment of assay performance.

For a ligand-binding assay, the measured response is expected to follow a curve that is sigmoidal in shape with a lower boundary (asymptote) near the minimum response and an upper asymptote near the maximum response. For data of this shape, the 4-parameter logistic model is generally acknowledged to be the reference model of choice for fitting [21]. In turn, this model forms the basis for the determination of a number of performance characteristics such as limit of detection (LOD), linear range and relative error (RE). Consequently, it is important to verify that the channel selected for analysis exhibits this expected response in order to validate the performance of the camera phone-based technique with that of the standard laboratory approach.

Fig. 2 shows response data for each color channel of the image sensor. It is clear that the $b$-channel provides the expected sigmoidal response and exhibits a sufficiently wide range of response (0.273) with low noise (0.015) for measurement (as indicated by the previously described SNR measure). In the case of the $g$-channel, the response is also sigmoidal in shape but does not exhibit a sufficiently large range (0.065). In fact, the $g$-channel is observed to be relatively insensitive to changes in sample concentration. Finally, the $r$-channel response data do not have the expected shape since a measurable response cannot be obtained at higher concentrations (i.e., 5.0 and $1.0 \mu \mathrm{g} / \mathrm{ml}$ ).

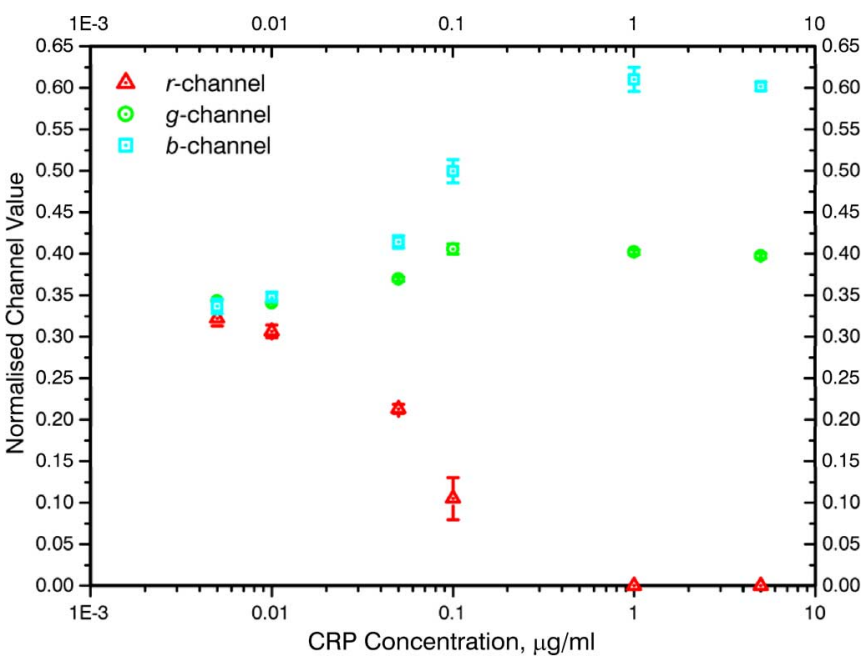

Fig. 2. Normalised color channel response as a function of CRP concentration (error bars $\pm 1 \sigma)$. (Blanks not shown).

This means that it may not be possible to reliably fit the data in this channel to a 4-parameter logistic model since this portion of the fitted model will be unbound.

\section{RESULTS \& CONCLUSION}

Following from the analysis of color channel performance, the response data of the $b$-channel was fitted to a 4-parameter logistic model (1) in order to assess system performance.

$$
y=P_{1}+\frac{P_{2}-P_{1}}{1+\left(\frac{x}{P_{3}}\right)^{P_{4}}}
$$

In this model, $y$ is the response (e.g., camera phone color channel value or laboratory instrument absorbance value), $x$ is the concentration, $\mathrm{P}_{1}$ is the upper asymptote, $\mathrm{P}_{2}$ is the lower asymptote, $\mathrm{P}_{3}$ is the model EC50 (the concentration corresponding to midway between the modeled estimates of minimum and maximum response) and $\mathrm{P}_{4}$ is the so-called slope factor.

Table II shows the response data obtained from the analysis of the 96 well plate using the standard laboratory instrument along with the $b$-channel response of the camera phone. Table III shows the fitted logistic model parameter values and the goodness of fit measures determined in each case. The camera phone data were found to exhibit excellent agreement $\left(\mathrm{R}^{2}=0.998\right)$ when fitted with the logistic model and closely agreed with the standard curve obtained using the laboratory instrument. Fig. 3 shows the standard curves obtained using the laboratory instrument and the $b$-channel of the camera phone along with the fitted 4-parameter logistic models.

The fitted 4-parameter logistic models were then used to estimate the limit of detection (LOD) for each method by determining the concentration $(x)$ at which the response $(y)$ is equal to

$$
\bar{y}_{\text {Blank }}+3 \sigma_{\text {Blank }}
$$


TABLE II

ELISA ANALYSIS RESULTS

\begin{tabular}{ccc}
\hline \hline & Laboratory Instrument & Camera Phone \\
\hline $\begin{array}{c}\text { Concentration } \\
\mu \mathrm{g} / \mathrm{ml}\end{array}$ & $\begin{array}{c}\text { Absorbance } @, 450 \mathrm{~nm} \\
( \pm 1 \sigma)\end{array}$ & $\begin{array}{c}b \text { - Channel Value } \\
( \pm 1 \sigma)\end{array}$ \\
\hline 5.000 & $1.0124 \pm 0.0478$ & $0.601 \pm 0.005$ \\
1.000 & $0.9884 \pm 0.1116$ & $0.610 \pm 0.140$ \\
0.100 & $0.5233 \pm 0.9673$ & $0.499 \pm 0.140$ \\
0.050 & $0.3081 \pm 0.0385$ & $0.414 \pm 0.070$ \\
0.010 & $0.1453 \pm 0.0067$ & $0.346 \pm 0.007$ \\
0.005 & $0.1379 \pm 0.0039$ & $0.336 \pm 0.008$ \\
Blank & $0.1094 \pm 0.0083$ & $0.333 \pm 0.011$ \\
\hline \hline
\end{tabular}

TABLE III

LOGISTIC MODEL PARAMETERS

\begin{tabular}{ccccccc}
\hline \hline & \multicolumn{4}{c}{ Model Parameters } & \multicolumn{2}{c}{ Fit } \\
\cline { 2 - 5 } & $\mathrm{P}_{1}$ & $\mathrm{P}_{2}$ & $\mathrm{P}_{3}$ & $\mathrm{P}_{4}$ & $\mathrm{R}^{2}$ & $\chi^{2}$ \\
\hline Laboratory & 1.0153 & 0.1220 & 0.1142 & 1.5889 & 0.999 & $1 \mathrm{E}-4$ \\
$\begin{array}{c}\text { Instrument } \\
\text { Camera } \\
\text { Phone }\end{array}$ & 0.6066 & 0.3359 & 0.0802 & 1.8872 & 0.998 & $3 \mathrm{E}-4$ \\
\hline \hline
\end{tabular}

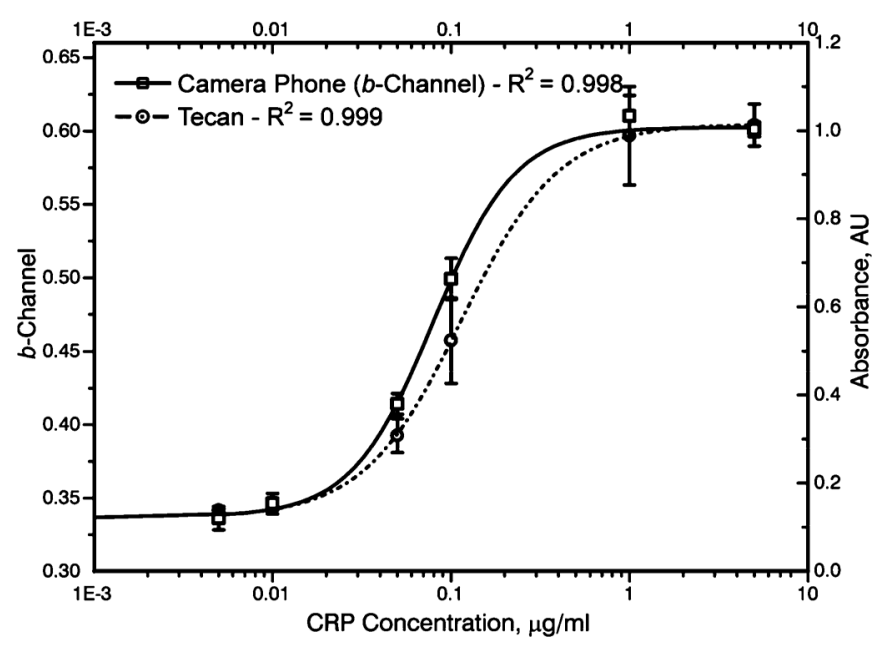

Fig. 3. hs-CRP ELISA standard curves obtained using camera phone and laboratory instrument (error bars $\pm 1 \sigma$ ). (Blanks not shown.)

where $\bar{y}_{\text {Blank }}$ is the average blank response and $\sigma_{\text {Blank }}$ is the blank response standard deviation. Using this approach, (2) is substituted for $y$ in (1) which is then rearranged for $x$ which becomes the LOD to give

$$
L O D=P_{3}\left(\frac{P_{2}-P_{1}}{\left(\bar{y}_{\text {Blank }}+3 \sigma_{\text {Blank }}\right)-P_{1}}-1\right)^{\frac{1}{P_{4}}} .
$$

The LOD for the camera phone-based method and laboratory instrument were determined to be $0.026 \pm 0.002 \mu \mathrm{g} / \mathrm{ml}$ $(1.035 \pm 0.079 \mu \mathrm{M})$ and $0.007 \pm 0.001 \mu \mathrm{g} / \mathrm{ml}(0.278 \pm$ $0.039 \mu \mathrm{M})$ CRP respectively. Using the method described in [22], the linear regions of the standard curves for the camera phone and laboratory instrument were found to be
$0.035-0.182 \mu \mathrm{g} / \mathrm{ml}(1.394-7.249 \mu \mathrm{M})$ and $0.043-0.302 \mu \mathrm{g} / \mathrm{ml}$ $(1.712-12.03 \mu \mathrm{M}) \mathrm{CRP}$ respectively. In both cases, the relative error (RE) in the linear regions was determined to be $<10 \%$. The unknown CRP concentration range was determined to be $0.040-0.055 \mu \mathrm{g} / \mathrm{ml}(1.593-2.191 \mu \mathrm{M})$ using the camera phone and $0.044-0.056 \mu \mathrm{g} / \mathrm{ml}(1.752-2.230 \mu \mathrm{M})$ using the laboratory instrument. These ranges compare favorably with the nominal unknown concentration of $0.055 \mu \mathrm{g} / \mathrm{ml}(2.191 \mu \mathrm{M})$.

These results clearly demonstrate that it is possible to perform quantitative analysis of a hs-CRP ELISA using a camera phone as an alternative low-cost optical detector. Moreover, this work shows that the basic imaging performance of a commercial camera phone can be used in conjunction with a suitably developed assay to enable quantitative measurement of CRP concentrations at clinically relevant levels with performance comparable to a standard laboratory technique. Clearly, this result has broad ramifications for the development of low-cost point-of-care (POC) technologies in general, however, specifically, it indicates a basic technology (i.e., optical detection platform) to enable remote quantitative monitoring of an important inflammatory biomarker used in the management of a number of chronic diseases (e.g., CVD, RA and IBD) may already be present in the patient's home.

As the global socio-economic burden of disease becomes more apparent, there is now growing interest in the development of connected- and mobile-health (mHealth) technologies to reduce this burden and enhance patient quality of life. In this regard, the innovative integration of technologies for the diagnosis and management of disease will play a crucial role. For example, the use of camera phones and microfluidic biochips has been demonstrated in the detection of cancer cells [23]. While this work represents an excellent technology demonstrator, the use of mobile phone-based technologies is more likely to find greater application in the monitoring of disease rather than in its diagnosis.

In future work, we will further develop and optimize the approach described here and investigate the use of microfluidic biochips and on-phone image analysis to provide a convenient, sample-to-result testing system for home-based monitoring of CRP. In this context, we have demonstrated that this is feasible given that the assay we describe has a relatively short incubation time and can be analysed in-situ using a camera phone. Furthermore, the elimination of the reaction stopping step reduces the number of reagents that must be stored on such a microfluidic chip and can reduce the complexity of the microfluidic architecture used. It is anticipated that the development of such a system could have a significant impact on the management of chronic inflammatory disease but also have broader application in other areas such as in the treatment of sepsis [24], prediction of heart disease and stroke [25], monitoring immune system response in cancer patients [26] as well as providing a means of monitoring wellness [27].

\section{REFERENCES}

[1] M. Pérez, M. Mera, J. R. Arias, B. G. Arza, C. E. Carleos, R. Muñiz, and C. de la Torre, "PocketELISA: A low-cost portable ELISA reader based on image analysis over PDA platform for clinical diagnose in medical veterinary," in Proc. IEEE Int. Symp. Industrial Electronics, 2008, pp. 939-943. 
[2] B. García, J. A. Baro, C. de la Torre, R. Muñiz, and M. A. Pérez, “A new low-cost and portable ELISA reader by using a photodiode matrix and Electroluminiscent (El) Lamps," in Proc. 19th IMEKO World Congress Fundamental and Applied Metrology Conf., 2009.

[3] P. Grosso, S. Carrara, C. Stagni, and L. Benini, "A low-cost point-ofcare system for parallel ELISA essays," in Proc. IEEE Biomedical Circuits and Systems Conf., 2009, pp. 269-272.

[4] N. A. Yusoff, N. Soin, and F. Ibrahim, "Lab-on-a-disk as a potential microfluidic platform for dengue NS1-ELISA," in Proc. IEEE Symp. Industrial Electronics and Applications, 2009, pp. 946-950.

[5] X. Y. Liu, C. M. Cheng, A. W. Martinez, K. A. Mirica, X. J. Li, S. T. Phillips, M. Mascareñas, and G. M. Whitesides, "A portable microfluidic paper-based device for ELISA," in Proc. IEEE 24th Int. Conf. Micro Electro Mechanical Systems, 2011, pp. 75-78.

[6] C. Yang, Y. Huang, B. L. Hassler, R. M. Worden, and A. J. Mason, "Amperometric electrochemical microsystem for a miniaturized protein biosensor array," IEEE Trans. Biomed. Circuits Syst., vol. 3, no. 3, pp. 160-168, 2009.

[7] O. Tigli, L. Bivona, P. Berg, and M. E. Zaghloul, "Fabrication and characterization of a surface-acoustic-wave biosensor in CMOS technology for cancer biomarker detection," IEEE Trans. Biomed. Circuits Syst., vol. 4, no. 1, pp. 62-73, 2010.

[8] L. Yao, P. Lamarche, N. Tawil, R. Khan, A. M. Aliakbar, M. H. Hassan, V. P. Chodavarapu, and R. Mandeville, "CMOS conductometric system for growth monitoring and sensing of bacteria," IEEE Trans. Biomed. Circuits Syst., vol. 5, no. 3, pp. 223-230, 2011.

[9] R. Copperwhite, C. McDonagh, and S. O'Driscoll, "A camera phonebased UV-dosimeter for monitoring the Solar Disinfection (SODIS) of water," IEEE Sensors J., vol. 12, no. 5, pp. 1425-1426, 2012.

[10] H. P. Ninh, Y. Tanaka, T. Nakamoto, and K. Hamada, "A bad-smell sensing network using gas detector tubes and mobile phone cameras," Sens. Actuators B, Chem., vol. 125, pp. 138-143, 2007.

[11] A. García, M. M. Erenas, E. D. Marinetto, C. A. Abad, I. de Orbe-Paya, A. J. Palma, and L. F. Capitán-Vallvey, "Mobile phone platform as portable chemical analyser," Sens. Actuators B, Chem., vol. 156, pp. 350-359, 2011.

[12] Y. Lu, W. Shi, J. Qin, and B. Lin, "Low cost, portable detection of gold nanoparticle-labeled microfluidic immunoassay with camera cell phone," Electrophor., vol. 30, pp. 579-582, 2009.

[13] A. W. Martinez, S. T. Phillips, E. Carrilho, S. W. Thomas III, H. Sindi, and G. M. Whitesides, "Simple telemedicine for developing regions: Camera phones and paper-based microfluidic devices for real-time, offsite diagnosis," Anal. Chem., vol. 80, pp. 3699-3707, 2008.

[14] D. Singh, "How can chronic disease management programmes operate across care settings and providers?," WHO, policy brief, 2008.

[15] T. A. Pearson et al., "Markers of inflammation and cardiovascular disease: Application to clinical and public health practice: A statement for healthcare professionals from the Centers for Disease Control and Prevention and the American Heart Association," Circulation, vol. 107, pp. 499-511, 2003.

[16] D. T. Felson et al., "American College of Rheumatology/European league against rheumatism provisional definition of remission in rheumatoid arthritis for clinical trials," Arthritis Rheum., vol. 63, pp. 573-586, 2011.

[17] M. Boirivant et al., "The clinical significance of serum $\mathrm{C}$ reactive protein levels in Crohn's disease. Results of a prospective longitudinal study," J Clin. Gastro., vol. 10, pp. 401-405, 1988.
[18] M. R. Laurent et al., "Circulating immune complexes, serum immunoglobulins, and acute phase proteins in psoriasis and psoriatic arthritis," Ann. Rheum. Dis., vol. 40, pp. 66-69, 1981.

[19] G. Finlayson, B. Schiele, and J. Crowley, "Comprehensive colour image normalization," in Proc. Eur. Conf. Computer Vision, 1998, pp. 475-490.

[20] H. R. Kang, Color Technology for Electronic Imaging Devices. Bellingham, WA, USA: SPIE, 1997.

[21] J. W. A. Findlay and R. F. Dillard, "Appropriate calibration curve fitting in Ligand binding assays," Amer. Assoc. Pharmaceut. Scientists J., vol. 8, pp. 260-267, 2007.

[22] J. L. Sebaugh and P. D. McCray, "Defining the linear portion of a sigmoidal-shaped curve: Bend points," Pharmaceut. Statist., vol. 2, pp. 167-164, 2003.

[23] S. Wang, X. Zhao, I. Khimji, R. Akbas, W. Qiu, D. Edwards, D. W. Cramer, B. Ye, and U. Demirci, "Integration of cell phone imaging with microchip ELISA to detect ovarian cancer HE4 biomarker in urine at the point-of-care," Lab Chip, vol. 11, pp. 3411-3418, 2011.

[24] P. Póvoa, "C-reactive protein: A valuable marker of sepsis," Intensive Care Med., vol. 28, pp. 235-243, 2002.

[25] P. M. Ridker, "C-reactive protein: A simple test to help predict heart attack and stroke," Circularion, vol. 108, pp. 81-85, 2003.

[26] B. Coventry et al., "CRP identifies homeostatic immune oscillations in cancer patients: A potential treatment targeting tool?," J. Translation. Med., vol. 7, p. 102, 2009.

[27] P. C. Kao, S. Shiesh, and T. Wu, "Serum C-reactive protein as a marker for wellness assessment," Ann. Clin. Lab. Sci., vol. 36, pp. 163-169, 2006.

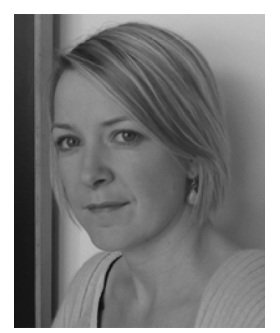

Cathy M. McGeough received the B.Sc. (Hons.) degree in biomedical science and the Ph.D. degree in immunogenetics from the University of Ulster (UU), Coleraine, Northern Ireland.

Currently, she is a Research Associate in Stratified Medicine at UU. Her research interests are inflammatory disease, personalized medicine, and diagnostics.

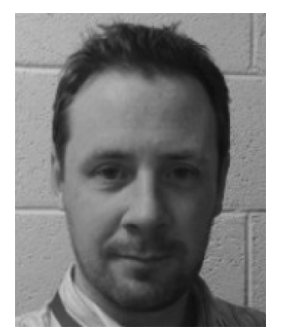

Stephen O'Driscoll (M'10) received the B.Sc. (Hons.) degree in applied physics and instrumentation, the M.Sc. degree in astrophysics, and the Ph.D. degree in environmental sensing from the Cork Institute of Technology, Cork, Ireland.

Currently, he is a Principal Investigator in the Biomedical Diagnostics Institute, Dublin City University, Dublin, Ireland, which he joined in 2010. In 2006, he joined the National Centre for Sensor Research at Dublin City University. His research interests include mHealth, point-of-care diagnostics, biosensor technologies, photonics, and signal/image processing. 